\title{
Analysis of the direct injury effector of oligodendroglia cells or myelin sheath in an experimental allergic encephalomyelitis model induced by the MOG35-55 peptide
}

\author{
XIANGYU ZHOU ${ }^{1}$, XIAOYONG $\mathrm{LI}^{2}$, MEINA FENG ${ }^{3}$, Q Z ZHANG $^{4}$ and ZHENDONG YANG ${ }^{5}$
}

\begin{abstract}
${ }^{1}$ Department of Neurology, The Affiliated First Hospital of Shizuishan, The Ningxia Medical University, Shizuishan, Ningxia 753200; ${ }^{2}$ Department of Pathology, Medical College, Hubei Polytechnic University, Huangshi, Hubei 435003;

${ }^{3}$ Department of Neurology, Wuhan Brain Hospital, Wuhan, Hubei 430010; ${ }^{4}$ Department of Neurology, Liyuan Hospital, Tongji Medical College, Huazhong University of Science and Technology, Wuhan, Hubei 430077; ${ }^{5}$ Department of Neurology, Wuhan No. 1 Hospital, Huazhong University of Science and Technology, Wuhan, Hubei 430022, P.R. China
\end{abstract}

Received May 27, 2014; Accepted July 28, 2015

DOI: $10.3892 / \mathrm{mmr} .2015 .4358$

\begin{abstract}
The aim of the present study was to investigate the possible role of cytotoxic T lymphocytes (CTL) and mononuclear macrophages in the pathogenic processes of experimental animals. To construct a chronic experimental allergic encephalomyelitis (EAE) model, an artificially synthesized myelin oligodendrocyte glycoprotein (MOG)35-55 peptide was used to induce C57BL/6 mice. Subsequently, the experimental animals were investigated at the level of their nervous function, and histopathological, immunohistochemical and fluorescence immunohistochemical experiments were performed at different time points following immunization. The expression of immune molecules and cytokines associated with the activation of the mononuclear macrophages and CTL during the different stages was assessed by western blotting and reverse transcription-quantitative polymerase chain reaction. As a result, the MOG35-55 peptide was identified as being successful at inducing C57BL/6 mice for the development of the EAE model. A modest level of mononuclear macrophage and lymphocyte infiltration was observed in the central nervous system (CNS), although no infiltration of neutrophils was observed. A sporadic flaky deletion of the myelin sheath was also identified. The activation and proliferation of mononuclear macrophages, including microglia
\end{abstract}

Correspondence to: Professor Qi Zhang, Department of Neurology, Liyuan Hospital, Tongji Medical College, Huazhong University of Science and Technology, 39 Lake Avenue, Wuhan, Hubei 430077, P.R. China

E-mail: zhangqi@mails.tjmu.edu.cn

Dr Zhendong Yang, Department of Neurology, Wuhan No. 1 Hospital, Huazhong University of Science and Technology, 215 Zhongshan Road Wuhan, Hubei 430022, P.R. China

E-mail: hbmy0503510@126.com

Key words: myelin oligodendrocyte glycoprotein, oligodendroglia cells, cytotoxic T lymphocyte, experimental allergic encephalomyelitis cells, was clearly demonstrated. Furthermore, the expression levels of major histocompatibility complex class I and II molecules and interleukin-12 in the brain, which is associated with the activation and proliferation of mononuclear macrophages, increased over the duration of the experiment compared with less pronounced changes in the expression levels of interferon (IFN) $-\gamma$, Fas and perforin in the CNS, which are associated with the function of CTL. The secretion of IFN- $\gamma$ in the spleen increased during the morbidity peak, however, any noticeable activation and proliferation of $\mathrm{CD}^{+} \mathrm{T}$ cells was absent. These results demonstrated that the induced immune response mediated by mononuclear macrophages made a more important contribution compared with CTL towards the pathological process of myelin sheath injury. Mononuclear macrophages are therefore, identified as being one of the most significant effector cell types to directly injure the myelin sheath in the CNS.

\section{Introduction}

Multiple sclerosis (MS) is an inflammatory autoimmune disease characterized by sporadic or multifocal demyelination in the white matter of the central nervous system (CNS), resulting in possible injury of the axons to differing degrees. Experimental allergic encephalomyelitis (EAE) is an inflammatory allergic disease affecting the CNS in a variety of sensitive experimental animals, and this is often used as an animal model of MS (1,2). Myelin oligodendrocyte glycoprotein (MOG) has been widely used in previous studies as the inducing antigen/immunogen for the EAE model. MOG is a transmembrane glycopeptide of $26-28 \mathrm{kDa}$, which is expressed in the myelin sheath membrane and on the outer surface of oligodendroglia cells $(3,4)$. Three identifiable epitopes exist in the extracellular region, and MOG35-55 peptide is one of the epitopes which causes encephalitis. MOG has been demonstrated to be the sole autoantigen, not only to induce the $\mathrm{T}$ cell response, but also to elicit the production of myelin sheath antibodies among the constitutive proteins of the myelin sheath (5), results which revealed the advantage of using MOG as the inducer for the establishment of the EAE 
model and its pathological characteristics (6). The EAE model of C57BL/6 mice induced by MOG approximates closely to human MS, with respect to the pathological and clinical manifestations (7-9), and therefore it is an ideal model to investigate the pathogenesis and therapeutic strategies of MS $(10,11)$.

The pathogenesis of MS remains to be fully elucidated, however, the best-supported hypothesis is that MS is a type of autoimmune disease of the myelin sheath in the CNS, in which autoreactive CD4+Th1 lymphocytes, and the cytokines they release, are the most important factors associated with humoral immunity. The impairment in function of the CNS is characterized by deletion of the myelin sheath, which is predominantly mediated by interferon (IFN)- $\gamma$ from activated $\mathrm{T}$ cells. This induces differentiation of the Th1 cells and the immune response targeted to the myelin sheath, leading to the development of the disease. An imbalance in the Th1/Th2 cell response, and either an inhibition of differentiation of the regulatory T cells (Treg cells) or an impairment of their function, are also likely to contribute towards the disease, even though autoreactive $\mathrm{CD} 4^{+} \mathrm{Th} 1$ lymphocytes and their released cytokines are thought to be primarily responsible as the causative agents (12-14). However, a number of biological phenomena, which are encountered during studies of MS/EAE, may not be explained by the predominant differentiation of the Th1 cells and the inhibition of differentiation or functional impairment of Treg cells under pathological conditions.

$\mathrm{CD} 4^{+} \mathrm{Th} 1$ and $\mathrm{CD}^{+} \mathrm{T}$ cells are unable to directly attack the target cells, and they contribute to inflammation and tissue injury predominantly by secreting cytokines, which promote the activation and proliferation of cytotoxic $\mathrm{T}$ lymphocytes (CTL), natural killer (NK) cells and phagocytes, and mediate the immune response of the effector cells. Th2 cells are also unable to cause direct injury of the target cells, and these mediate the humoral immune response predominantly by stimulating B lymphocytes to produce antibodies. Activated $\mathrm{CD}^{+} \mathrm{T}$ lymphocytes differentiate into $\mathrm{CD}^{+}$effector $\mathrm{T}$ cells and CTL. It is possible that oligodendroglia cells are attacked by activated CTL and macrophages, since the expression levels of the major histocompatibility complex (MHC) class I molecules are upregulated in oligodendroglia cells during inflammation. The immunocytes, which elicit direct myelin sheath injury of the CNS, are possibly those among the CTL or macrophage systems. The present study focused on an examination of the immune response changes of CTL and mononuclear macrophages using an EAE model, and aimed to ascertain which effector cells led to direct myelin sheath injury of the CNS by comparing the roles of CTL and mononuclear macrophages during the progression of EAE.

\section{Materials and methods}

Immunogens. Murine recombinant MOG35-55 peptide was artificially synthesized (Sigma-Alrich, St. Louis, MO, USA; purity, >98\%). The amino acid sequence of the peptide was MEVGWYRSPFSRVVHLYRNGK, with a molecular mass of 2,582.0 Daltons.

Experimental animals. A total of 80 healthy female wild-type C57BL/6 mice, aged 8-10 weeks and weighing 18-22 g, were used in the present study. The mice were provided by the
Experimental Animal Center (Wuhan University, Wuhan, China) and fed on nutritional foodstuff in individual cages. The feeding environment was at room temperature $\left(18-25^{\circ} \mathrm{C}\right)$, with a relative humidity of 50-60\%, and $12 \mathrm{~h}$ day/night cycle lighting. The mice were numbered and grouped following adaptive feeding for 1-2 weeks. The study was approved by the Ethics Committee of Huazhong University of Science and Technology (Wuhan, China).

Animal grouping and establishment of the EAE model. The 80 C57BL/6 mice were divided randomly into a blank control group and the EAE group, with 40 mice in each group. The control group animals were injected with 2 mg Complete ${ }^{\text {TM }}$ Freund's adjuvant (Sigma-Aldrich, St. Louis, MO, USA) subcutaneously at two points of the inguinal groove for each mouse and $200 \mathrm{ng}$ pertussis toxin (Sigma-Aldrich) was subsequently injected into the abdominal cavity on the day of immunization and $48 \mathrm{~h}$ afterwards. Administration of the OG35-55 peptide and immunological adjuvant were used to establish the chronic EAE animal model in the EAE group: $0.2 \mathrm{ml}$ mixed emulsion was injected subcutaneously at two points of the inguinal groove for each mouse, which contained $250 \mu \mathrm{g}$ artificially synthesized MOG35-55 peptide and $2 \mathrm{mg}$ Complete ${ }^{\mathrm{TM}}$ Freund's adjuvant (volume ratio, 1:1). The mice were subsequently injected with $200 \mathrm{ng}$ pertussis toxin into the abdominal cavity on the day of immunization and $48 \mathrm{~h}$ afterwards. The behavior and activities of the animals were assessed daily prior to the completion of the experiment, on day 30, with the day of immunization being set as day 0 . The severity of clinical nervous symptoms of the EAE group mice were assessed according to the Benson scoring standard (15). A total of eight mice were sacrificed in the control and EAE groups on days $0,7,14,21$ and 30 , and brain and spleen tissues were extracted for further examination. The mice were anesthetized with $3 \mathrm{ml} / \mathrm{kg}$ chloral hydrate (Sigma-Aldrich), following which the thoracic cavity was opened in order to expose the heart. A needle was inserted from the apex cordis to the aorta, and the right auricle was sectioned. A total of $0.9 \%$ normal saline (NS; $300 \mathrm{ml}$ ) was perfused until the perfusate was transparent, followed by perfusion of $50 \mathrm{ml} 4 \%$ paraformaldehyde (Servicebio Technology Co., Ltd, Wuhan, China).

The scoring standard was applied as follows: 0 points, no symptoms; 1 point, tail inertia or postscript crouch gait accompanied by powerful tail; 2 points, jump crouch gait accompanied by tail inertia (ataxia); 2.5 points, ataxia accompanied by partial paralysis of a single limb; 3 points, complete paralysis of a single limb; 3.5 points, complete paralysis of a single limb accompanied by partial paralysis of another limb; 4 points, complete paralysis of a couple of limbs; 4.5 points, paralysis of four limbs; 5 points, death.

Histological examination. The abdominal skin of the experimental mice was cut from the upper part along a curved line to each side following anesthetization by injection of $10 \%$ chloral hydrate $(3 \mathrm{ml} / \mathrm{kg})$ into the abdominal cavity. The peritoneum and costal bone on each side was carefully incised to completely expose the thoracic cavity. A perfusion needle was fixed, and inserted from the cardiac apex up to the aorta. The right auricular appendix was cut and perfused with 


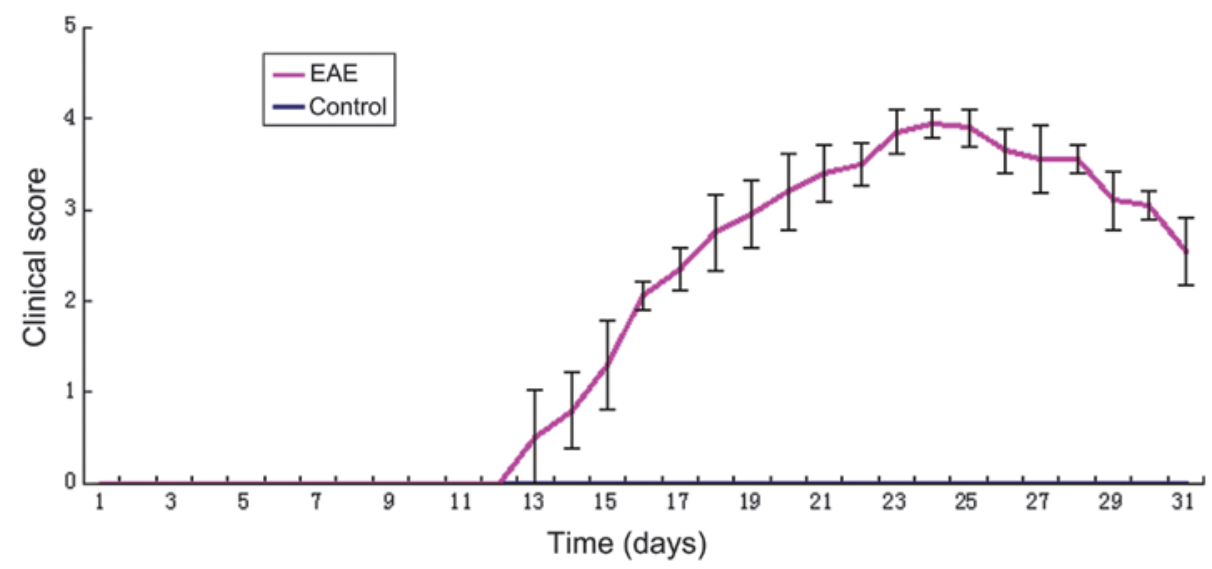

Figure 1. Clinical function scores of the experimental mice. The data are expressed as the mean \pm standard error of the mean. EAE, experimental allergic encephalomyelitis.

$\sim 300 \mathrm{ml} 0.9 \%$ pre-cooled NS rapidly until clear fluid flowed out. This was followed by initially rapid and then slow perfusion of $500 \mathrm{ml} 4 \%$ paraformaldehyde solution. An incision was made to the scalp of the mice and the cranial bone was removed. The spleen was removed from the abdominal cavity following careful extraction of the whole brain tissue, and this was rinsed briefly with NS prior to placement in $4 \%$ paraformaldehyde fixation solution. The tissue was paraffin-embedded and sliced, followed by hematoxylin-eosin (H\&E; Sigma-Aldrich) staining, Luxol Fast Blue (LFB; Sigma-Aldrich) staining and fluorescence immunohistochemistry (IHC). Antibodies, together with their dilutions, were as follows: Mouse anti-mouse IFN- $\gamma$ monoclonal immunoglobulin (Ig)G (cat. no. ab22543), 1:100; goat anti-mouse Iba-1 polyclonal IgG (cat. no. ab107159), 1:100; rabbit anti-mouse CD8 polyclonal IgG (cat. no. ab191905), 1:100; rabbit anti-mouse ki67 polyclonal IgG (cat. no. ab15580), 1:100 (Abcam, Cambridge, UK).

Western blotting. The rat brains were harvested and homogenized at $4^{\circ} \mathrm{C}$ using a Teflon glass homogenizer in $50 \mathrm{mM}$ Tris- $\mathrm{HCl}$ (pH 7.4), $150 \mathrm{mM} \mathrm{NaCl}, 10 \mathrm{mM} \mathrm{NaF}, 1 \mathrm{mM} \mathrm{Na}_{3} \mathrm{VO}_{4}$, $10 \mathrm{mM} \beta$-mercaptoethanol, $5 \mathrm{mM}$ EDTA, $2 \mathrm{mM}$ benzamidine, $1.0 \mathrm{mM}$ phenylmethanesulfonyl fluoride, $5 \mathrm{mg} / \mathrm{ml}$ leupeptin, $5 \mathrm{mg} / \mathrm{ml}$ aprotinin and $2 \mathrm{mg} / \mathrm{ml}$ pepstatin (Sigma-Aldrich). Three volumes of the homogenized samples were subsequently added to one volume of the extracting buffer $(200 \mathrm{mM}$ Tris-HCl, pH 7.6, 8\% SDS, 40\% glycerol; Sigma-Aldrich). Protein concentrations were determined using a bicinchoninic acid kit (Pierce Biotechnology, Inc., Rockford, IL, USA) and between 9 and $11 \mathrm{mg} / \mathrm{ml}$ was used. A final concentration of $10 \%$ mercaptoethanol and $0.05 \%$ bromophenol blue (Sigma-Aldrich) were then added, and the samples were boiled in a water bath for $10 \mathrm{~min}$. The boiled samples were separated by $10 \%$ SDS-PAGE (Sigma-Aldrich) and the separated proteins were transferred onto nitrocellulose membranes (GE Healthcare Life Sciences, Little Chalfont, UK). The membranes were subsequently blocked with $5 \%$ nonfat milk dissolved in Tris-buffered saline (TBS)-Tween-20 (50 mM Tris-HCl, pH 7.6,

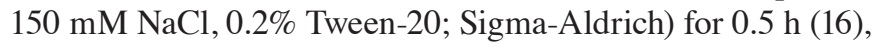
followed by an incubation with primary antibodies (1:200) at $25^{\circ} \mathrm{C}$ for $0.5 \mathrm{~h}$. The primary antibodies were as follows: Mouse anti-mouse monoclonal IgG GAPDH (cat. no. ab9482), rabbit anti-mouse polyclonal IgG MHC-I (cat. no. ab93364), rat anti-mouse monoclonal IgG MHC-II (cat. no. ab139365) and rat anti-mouse monoclonal IgG L-12 (cat. no. ab80682) (Abcam). The membranes were washed with TBS-Tween-20, and then incubated with horseradish peroxidase-conjugated anti-mouse or anti-rabbit IgG antibodies $(1: 15,000)$ for $1 \mathrm{~h}$ at room temperature. After a final wash with TBS-Tween-20, the grey scale of the blots was analyzed using an Odyssey Infrared Imaging system (cat. no. 9120; LI-COR Biosciences, Lincoln, NE, USA).

Reverse transcription-quantitative polymerase chain reaction $(R T-q P C R)$. For RT-qPCR, mice tissue during all the experimental stages was selected and the total RNA was extracted using TRIzol ${ }^{\circledR}$ reagent (Invitrogen Life Technologies, Carlsbad, CA, USA), according to the manufacturer's instructions. The cDNA $(5 \mu \mathrm{g})$ was reverse-transcribed and synthesized using a Prime Script First Strand cDNA Synthesis kit (cat. no. D6110A; Takara Bio, Inc., Beijing, China) under the conditions of $37^{\circ} \mathrm{C}$ for $15 \mathrm{~min}$ and $85^{\circ} \mathrm{C}$ for $5 \mathrm{sec}$. The gene products were subsequently amplified by RT-qPCR (CFX Connect ${ }^{\mathrm{TM}}$ Optics Module; Bio-Rad Laboratories, Inc.), according to the following method. The reaction system comprised $5 \mu \mathrm{l} \mathrm{SYBR}$ Green mix (Sigma-Aldrich), $0.3 \mu 1$ the upstream and downstream primers, $1 \mu \mathrm{l}$ cDNA and $3.4 \mu \mathrm{l}$ RNase-free water. A total of 40 cycles were used in the program $\left(95^{\circ} \mathrm{C}\right.$ for $3 \mathrm{~min}, 95^{\circ} \mathrm{C}$ for $10 \mathrm{sec}, 60^{\circ} \mathrm{C}$ for $30 \mathrm{sec}$ ), and the dissolving curve was examined at $65-90^{\circ} \mathrm{C}$. The sequences of the primers used were as follows: $\beta$-actin, upstream: 5'-CTGAGAGGGAAATCGTGCGT-3' and downstream: 5'-CCACAGGATTCCATACCCAAGA-3'; Fas, upstream: 5'-CACCCTGACCCAGAATACCAAG-3'; and downstream: 5'-AGGCGATTTCTGGGACTTTGT-3'; perforin, upstream: 5'- CACGCATGATCTGCTCTTCG-3' and downstream: 5'-CGCTTCGGGTTCTGTTCTTC-3'.

Statistical analysis. SPSS 18.0 software (IBM, SPSS, Chicago, IL, USA) was used for statistical analysis of the experimental data. The data are expressed as the mean \pm standard error of the mean. Student's t-test was used to compare the differences between pairs of groups, and one-way analysis of variance was used to compare several samples. $\mathrm{P}<0.05$ was considered to indicate a statistically significant difference. 

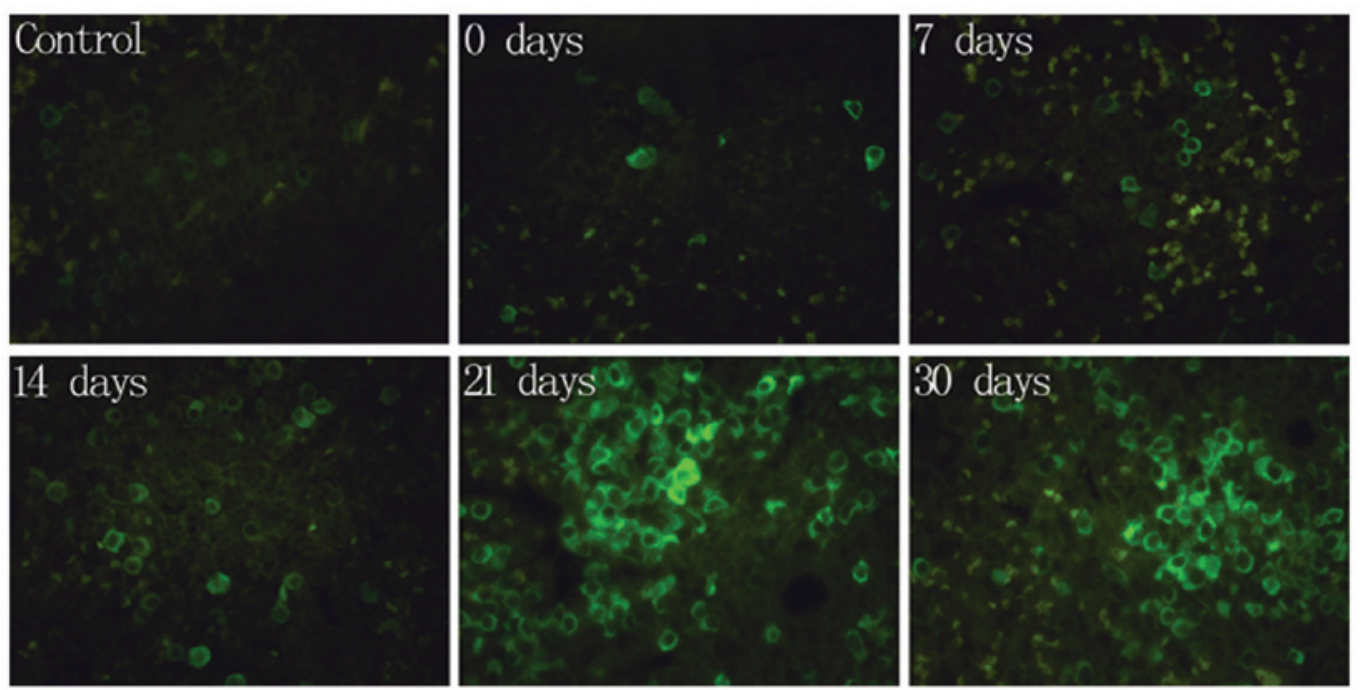

Figure 2. Fluorescence immunohistochemistry of IFN- $\gamma$ in the spleen of the experimental mice (magnification, $\mathrm{x} 200$ ), measured over the 30 day duration of the experiment. The green coloration indicated the presence of IFN- $\gamma$. IFN, interferon.

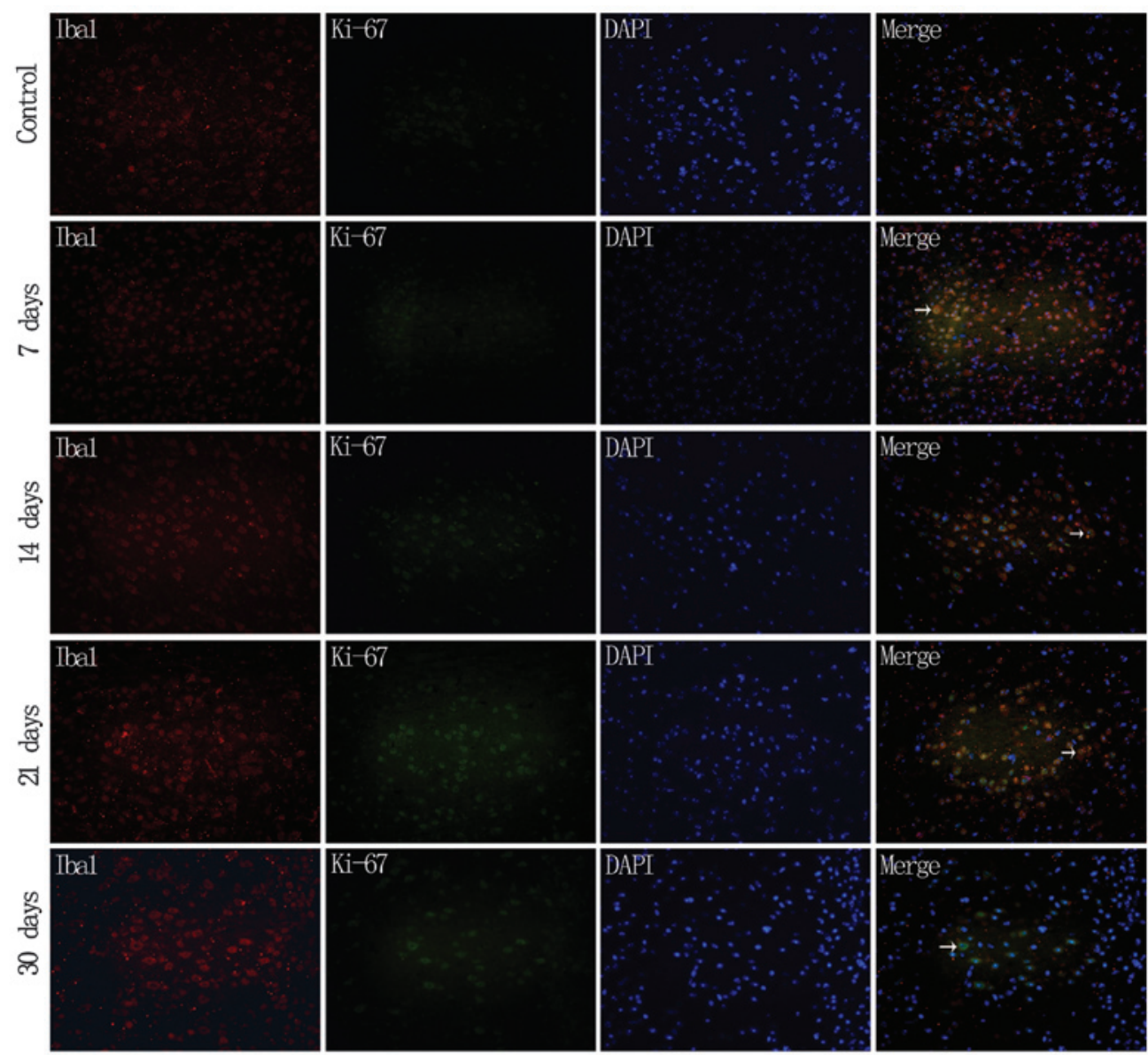

Figure 3. Double fluorescence immunohistochemistry of Iba1 and Ki-67 in the brain of the experimental mice (magnification, x200). The merged images are presented in the panels on the right. DAPI, 4',6-diamidine-2'-phenylindole dihydrochloride.

\section{Results}

Establishment of the animal model. The EAE model of C57BL/6J mice was successfully induced by injection of the MOG35-55 peptide and immunological adjuvant. As shown in Fig. 1 , the experiment was terminated on day 30 , and all mice in the control group were free of the disease. The disease affected all mice in the EAE model group, with the exception of those which were sacrificed during the early stage. The mice in the EAE group were free of the disease up to 11 days following immunization, at which time certain mice were affected by the disease in rapid succession, between days 12 and 14, with 

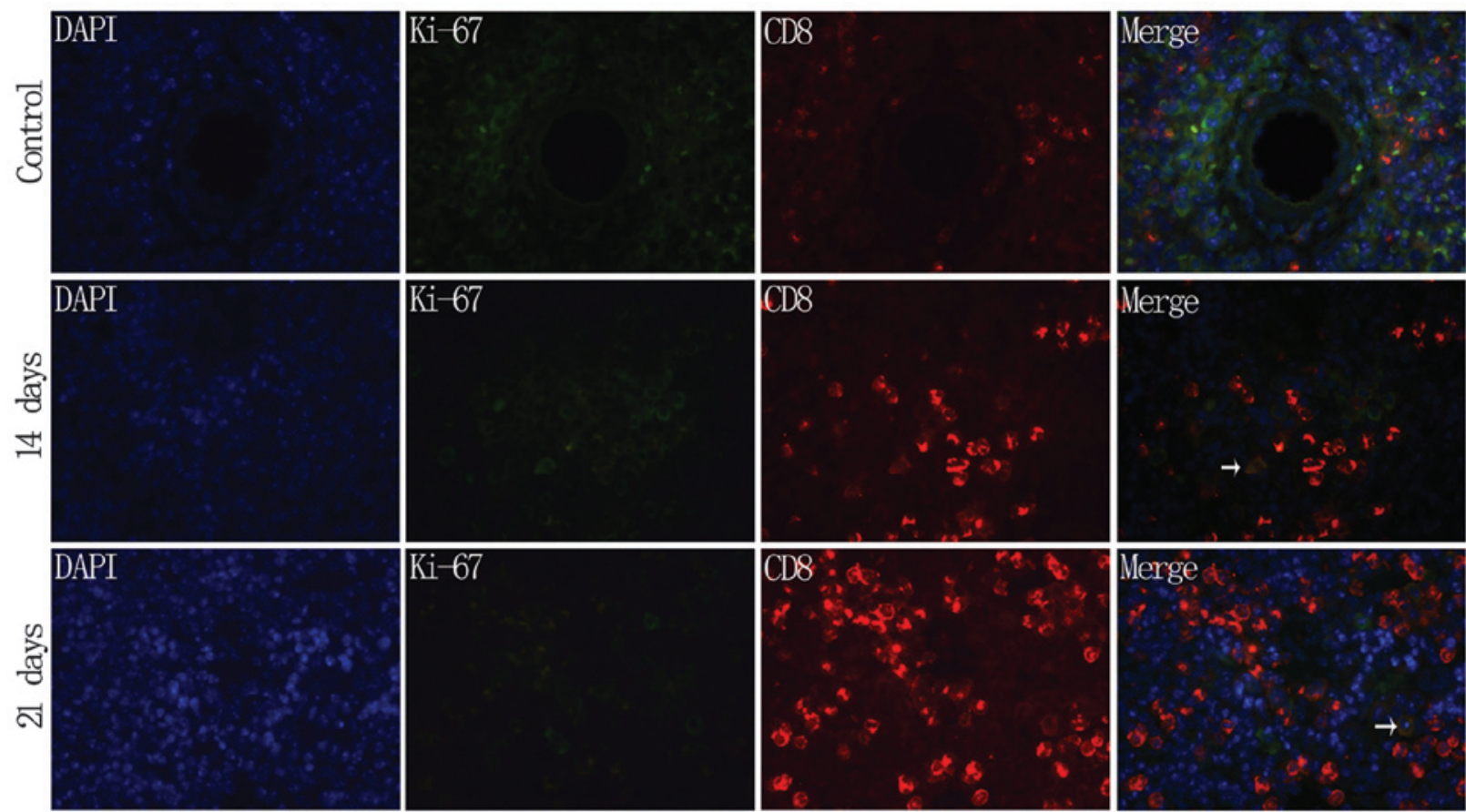

Figure 4. Double fluorescence IHC of CD8 and Ki-67 in the spleen of the experimental mice (magnification, x200). The merged images are presented in the panels on the right. DAPI, 4',6-diamidine-2'-phenylindole dihydrochloride.

A

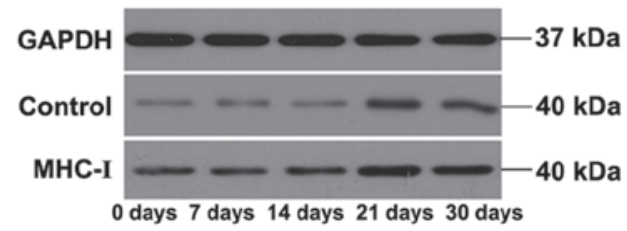

C

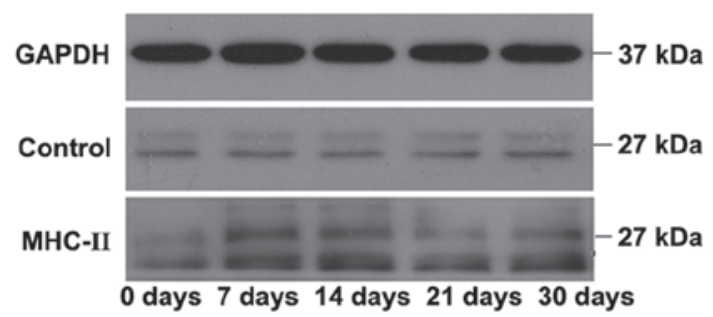

B

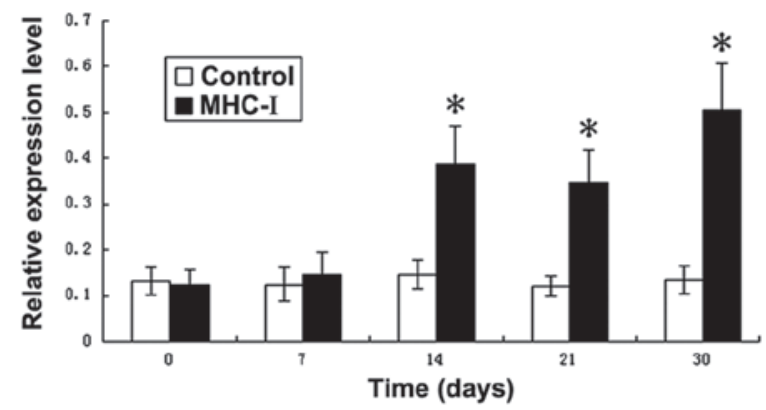

D
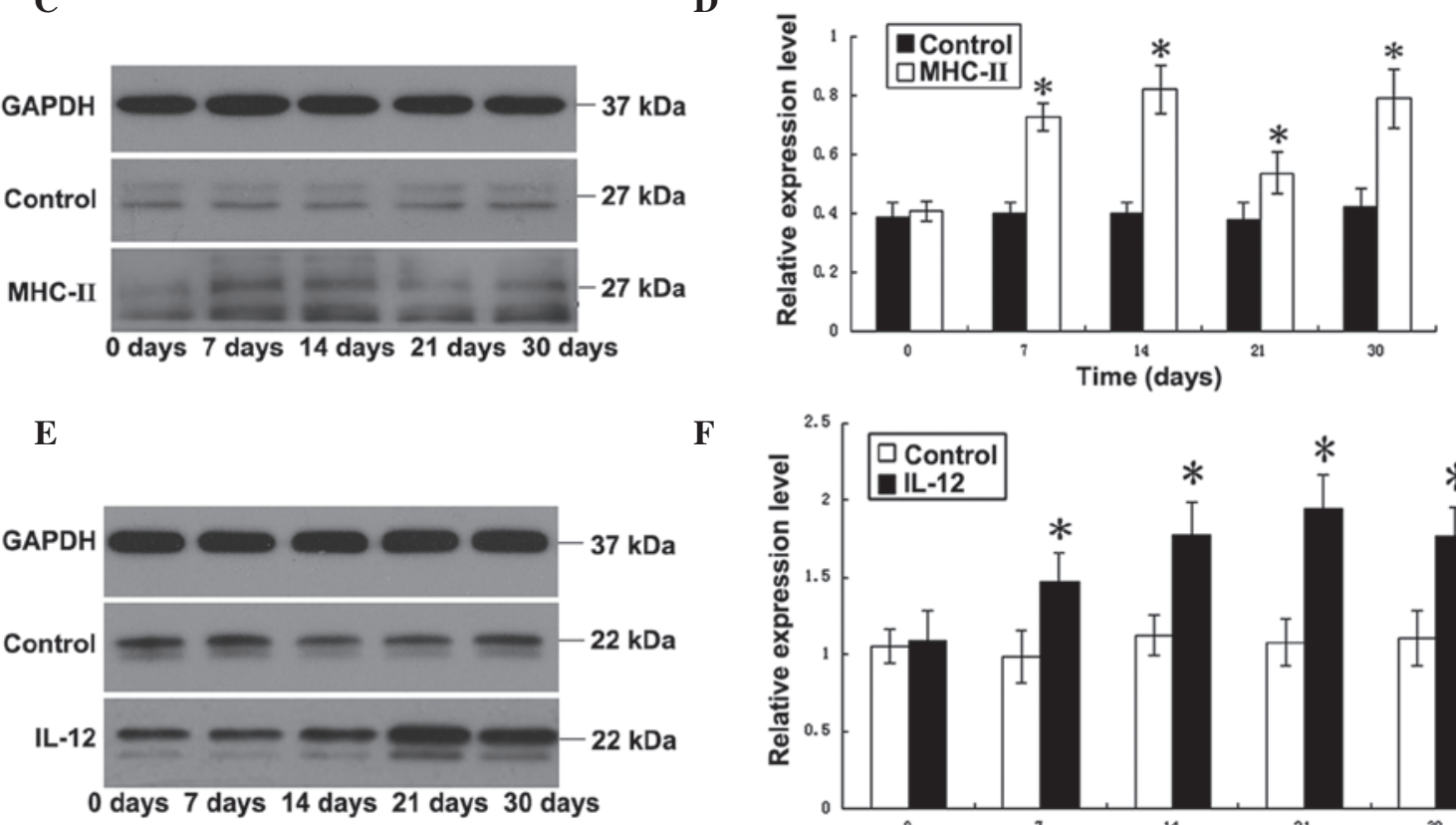

$\mathbf{F}$

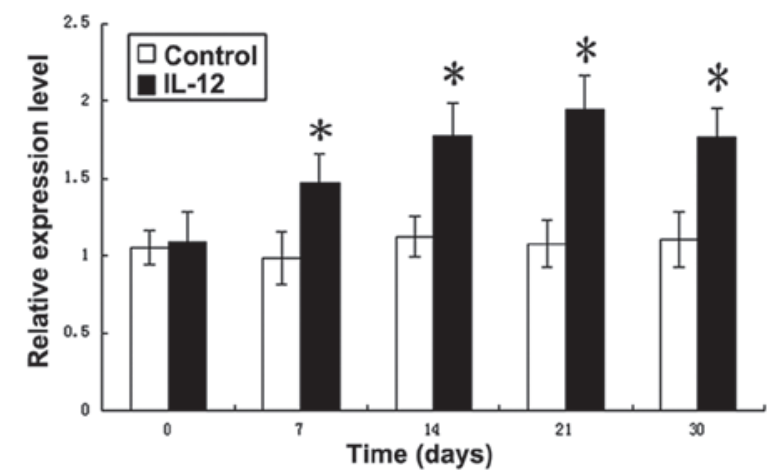

Figure 5. Examination of the levels of (A and B) MHC I, (C and D) MHC II and (E and F) IL-12 in the brain of the experimental mice during all stages. The panels on the left demonstrate the western blot analyses. The quantified data are revealed in the panels on the right ("P<0.05, compared with the control group). GAPDH, glyceraldehyde-3-phosphate dehydrogenase; IL, interleukin; MHC, major histocompatibility complex. 
A

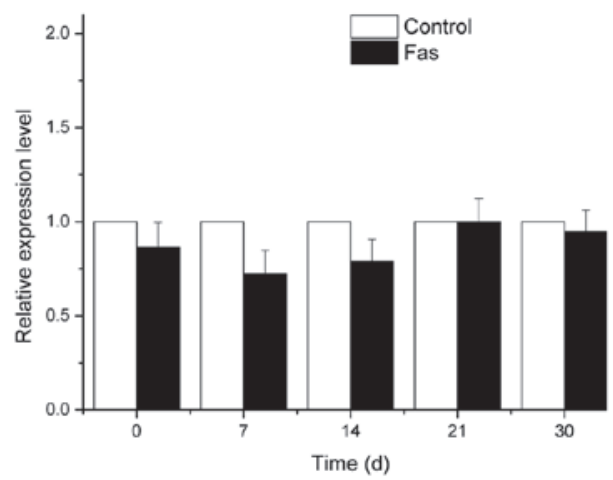

B

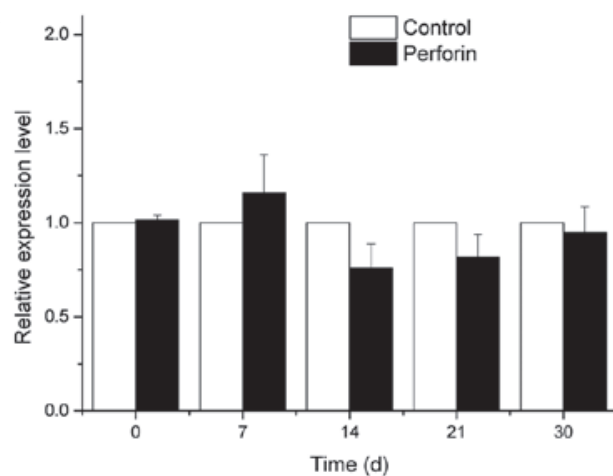

Figure 6. Changes in the mRNA expression levels of (A) Fas and (B) perforin in the brain of the mice in the experimental allergic encephalomyelitis model group (black bars) and the control group (white bars) at the different experimental stages. d, days.

clinical scores of $1-2$. The morbidity reached its peak during days 20-24, with scores of 2.5-4.5. The clinical symptoms of certain mice were alleviated following survival for 3-4 days, and their scores declined. However, the symptoms persisted and the disease was able to enter the chronic stage.

Histological changes in the brain of the experimental mice. The pathological condition of the mice was monitored throughout the 30 day course of the experiment. The brain tissue in each group appeared normal to the naked eye, however, a noticeable level of congestion and edema were observed in the spleen of the diseased mice in the EAE group. HE staining and LFB staining of the mice brain in the control group revealed no abnormal change. In the EAE group, a modest level of monocyte and lymphocyte infiltration in the brain was observed, as the disease progressed. LFB staining revealed a loosening of the white matter, discontinuity, breaking and vacuole changes in the myelin sheath, and a white and flaky deletion of the myelin sheath in certain regions, conforming to the features of the chronic EAE mode (i.e. prominent myelin sheath destruction and little apparent inflammatory cell infiltration).

Fig. 2 shows the results of the fluorescence IHC experiment of IFN- $\gamma$ in the spleen and brain of the experimental mice. A marginal quantity of green IFN- $\gamma$ fluorescence was observed by fluorescence IHC of IFN- $\gamma$ in the spleen of the control group, as revealed in Figs. 2-4. In the EAE group, green fluorescence was observed in the spleen on day 21 due to an abundant production of IFN- $\gamma$ by lymphocytes, indicating that the T lymphocytes were activated. The noticeable fluorescence coloration remained visible until day 30 . However, no positive identification of IFN- $\gamma$ was measured by fluorescence IHC in the brain tissue, with the exception of a very slight quantity of green IFN- $\gamma$ fluorescence around the ventricle on day 21 (data not shown).

Double fluorescence IHC of Iba1 and ki-67 in the brain of the experimental mice was subsequently performed (Fig. 3). No indication of proliferation of the Iba1-positive cells in the control group was evident, and a slight double fluorescence was visible on day 7 after immunization, which remained apparent up to day 14. On conclusion of the experiment at day 30, the proliferation reaction of Iba 1-positive cells remained visible.

Subsequently, a double fluorescence IPC experiment was performed with the anti-CD8 and anti-ki-67 antibodies in the spleen of the experimental mice. As revealed in Fig. 4, a slight and sporadic proliferation reaction of the $\mathrm{CD} 8^{+} \mathrm{T}$ lymphocytes was visible on days 14 and 21 after immunization, whereas no proliferation was observed during other stages, indicating that the majority of the activated and proliferating lymphocytes were not $\mathrm{CD}^{+} \mathrm{T}$ lymphocytes.

EAE altered the expression of the associated proteins. As revealed in Fig. 5, the expression level of MHC I molecules in the brain tissue of mice in the EAE group increased significantly on day 14, and the higher levels of MHC I persisted until day 30 ( $\mathrm{P}<0.05$, compared with the control group). The results indicated that the expression of MHC I molecules increased with the onset of the disease, and the increased levels were maintained at the higher level without a decline. The expression levels of the MHC II molecules in mice in the EAE group increased significantly by day 7 following the onset of the disease, reached a peak on day 14, exhibited a decline on day 21 and subsequently rose again on day 30 , ( $\mathrm{P}<0.05$, compared with the control group). The secretion of IL-12 in the brain started to increase on day 7 and achieved its maximum level by day 21 , maintaining a higher level up until day 30. The increases compared with the control group were significant $(\mathrm{P}<0.05)$; however, the level of IL-12 during the chronic stage of the disease was lower compared with that during the peak stage $(\mathrm{P}<0.05)$.

EAE did no affect the mRNA expression levels of Fas and perforin. Changes in the mRNA expression levels of Fas and perforin were examined in the brain of the mice in the EAE and control groups during the different stages of the experiment. The differences identified were not significant $(\mathrm{P}>0.05$; Fig. 6).

\section{Discussion}

It would appear that almost all the known immunocytes are involved in processes associated with inflammation and demyelination in the CNS, although the exact mechanisms of various immunocytes and their secreted cytokines in MS development remain to be fully elucidated (17-20). Antigens themselves are not the direct causative agents of disease during the immune response: Immunocytes, and molecules that are activated by the antigen directly or indirectly, are the direct cause. It is generally accepted that injury of the CNS, which 
is characterized predominantly by demyelination, is mediated by IFN- $\gamma$, a strong proinflammatory cytokine, which elicits the immune response of myelin sheath by inducing differentiation of the Th1 cells. However, a study investigating IFN- $\gamma$ and its receptor with gene-knockout animals revealed that EAE still occurred, and that the gene-knockout animals were more adversely affected by the disease (21). This demonstrates that MS/EAE may be caused by several types of pathological mechanisms working in concert, and innate immunity, the specific immune response and other regulatory mechanisms of the immune response may be involved.

Cellular functions become diversified following lymphocyte activation during the effector stage. $\mathrm{T}_{0}$ lymphocytes differentiate into $\mathrm{CD}^{+}$and $\mathrm{CD}^{+} \mathrm{T}$ lymphocytes and Treg cells. Each type of $\mathrm{T}$ lymphocyte then further differentiates into the terminal immune effector cells. CTL can attack the host cells directly, however, Th cells, Treg cells and CD8 ${ }^{+}$ T effector lymphocytes (together with B lymphocytes, another type of humoral immunocyte) only regulate the direction and extent of the immune response by secreting cytokines or antibodies, which act further on macrophages and CTL, without being able to attack the host cells directly. At present, macrophages and CTL are known to cause direct injury of oligodendroglia cells and the myelin sheath.

The macrophages, which are predominately featured in the MS/EAE immune response are mononuclear macrophages and neutrophils. In the present study, no infiltration and aggregation of neutrophils was identified in the CNS, indicating that the major pathogenic macrophages may be oligodendroglia cells in the CNS and/or peripheral mononuclear macrophages. MHC molecules are not constitutively expressed by oligodendroglia cells, although MHC I molecules can be upregulated under pathological conditions, including inflammation, whereas the expression of MHC II molecules by oligodendroglia cells does not occur under any conditions. This further demonstrated that CD4 ${ }^{+}$Th lymphocytes and Treg cells do not directly attack oligodendroglia cells.

CTL are restricted by MHC I molecules when killing the target cells, and sensitized CTL cells are able to continuously act on other target cells, which carry an identical antigen following killing of the target cells, demonstrating that the effects of CTL are self-propagating. A number of previous studies revealed that the role of CTL in the occurrence and development of MS/EAE is not negligible, however, it is controversial (22-25). Additionally, it was revealed that $\mathrm{CD}^{+}$Treg cells express specificity for their target organs, which may lead to the direct killing of the activated immune response cells, or produce cytokines, including TGF- $\beta$ and IL-10, under the action of IFN- $\gamma$, fulfilling the role of immune suppression (26). CTL elicit their effects predominantly via the perforin and Fas/Fas L route. In the present study, changes identified for Fas, perforin and IFN- $\gamma$ during the occurrence and development of chronic EAE were not significant, and since they are associated with CTL function in the brain, no appreciable changes were observed in the levels of these effector molecules of CTL, which are capable of killing the target cells directly. Nevertheless, the expression of the MHC I and MHC II molecules, which are associated with the antigen-presenting function of mononuclear macrophages, were upregulated, indicating that there is also an increase in the expression levels of MHC I molecules in the CNS, despite the evidence that $\mathrm{T}$ lymphocyte activation is marked by IFN- $\gamma$ production in spleen. These results fail to demonstrate that CTL enhance the immune effect of MHC I positive cells in the CNS via the perforin and Fas/Fas L route.

Mononuclear macrophages are a type of antigen presenting cell (APC), which may also cause injury of the myelin sheath in CNS by phagocytosis, dissolution, and even possibly direct attack, in addition to the induction of the immune response by presenting antigens. IL-12 is a type of cytokine specifically produced by APC cells, including mononuclear macrophages and dendritic cells. Activated microglia cells are one of the predominant sources of IL-12 in the CNS. IL-12 may activate $\mathrm{NK}$ cells in innate immunity, however, its major function, identical to IFN- $\gamma$, is to promote the differentiation of Th0 cells into Th1 cells. In the present study, the expression levels of IL-12 changed markedly in animals in the EAE group throughout the course of the experiment, whereas the immunofluorescence studies revealed an absence of any appreciable increase in IFN- $\gamma$ secretion in the brain, indicating that the degree of T lymphocyte activation was not high. Given that the effects of IL-12 secreted by mononuclear macrophages on the $\mathrm{CNS}$ are mediated earlier than those of IFN- $\gamma$, and the effect of IL-12 in promoting Th cell differentiation and regulating antibody production of B lymphocytes is more marked compared with IFN- $\gamma$, mononuclear macrophages exert a more important role compared with CTL in the progression of EAE.

In conclusion, the effector cells causing direct injury to the myelin sheath in the CNS may be mononuclear macrophages other than CTL, including microglia cells, although it was not determined whether the Iba 1-positive cells are mononuclear macrophages migrating from peripheral tissues, or microglia cells residing in the brain. A noticeable activation and proliferation of mononuclear macrophages containing microglia cells during the course of EAE, and the induced immune response fulfils a more important role compared with CTL during the pathological process of myelin sheath injury. Therefore, mononuclear macrophages are one of the most important effector cells causing direct injury of the myelin sheath in the CNS.

\section{Acknowledgements}

This study was supported by the National Program on Key Basic Research Project of China (973 Program; no. 2012CB722401), and the National Natural Science Foundation of China (nos. 81030051 and 21177046).

\section{References}

1. Bradl $\mathrm{M}$ and Linington $\mathrm{C}$ : Animal model of demyelination. Brain Pathol 6: 303-311, 1996.

2. Polman $\mathrm{CH}$, Dijkstra CD, Sminia $\mathrm{T}$ and Koetsier JC: Immunohistological analysis of macrophages in the central nervous system of Lewis rats with experimental autoimmune encephalomyelitis. J Neuroimmunol 11: 215-21, 1986.

3. Mendel 1, Keriero de Rosbo N and Ben-Nun A: A myelin oligodendrocyte glycoprotein peptide induces typical chronic experimental autoimmune encephalomyelitis in $\mathrm{H}-2 \mathrm{~b}$ mice: Fine specificity and $\mathrm{T}$ cell receptor $\mathrm{V}$ beta expression of encephalitogenic T cells. Eur J Immunol 25: 1951-1959, 1995.

4. Pál E, Yamamura T and Tabira T: Autonomic regulation of experimental autoimmune encephalomyelitis in IL-4 knockout mice. J Neuroimmunol 100: 149-155, 1999. 
5. Baumann N and Pham-Dinh D: Biology of oligodendrocyte and myelin in the mammalian central nervous system. Physiol Rev 81: 871-927, 2001.

6. Wang H, Munger KL, Reindl M, O'Reilly EJ, Levin LI, Berger T and Ascherio A: Myelin oligodendrocyte glycoprotein antibodies and multiple sclerosis in healthy young adults. Neurology 71: 1142-1146, 2008

7. Roscoe WA, Welsh ME, Carter DE and Karlik SJ: VEGF and angiogenesis in acute and chronic MOG((35-55)) peptide induced EAE. J Neuroimmunol 209: 6-15, 2009.

8. Berard JL, Wolak K, Fournier S and David S: Characterization of relapsing-remitting and chronic forms of experimental autoimmune encephalomyelitis in C57BL/6 mice. Glia 58: 434-445, 2010.

9. Slavin A, Ewing C, Liu J, Ichikawa M, Slavin J and Bernard CC: Induction of a multiple sclerosis-like disease in mice with an immunodominant epitope of myelin oligodendrocyte glycoprotein. Autoimmunity 28: 109-120, 1998.

10. Kanamori M, Kawaguchi T, Nigro JM, Feuerstein BG, Berger MS, Miele L and Pieper RO: Contribution of Notch signaling activation to human glioblastoma multiforme. J Neurosurg 106: 417-427, 2007.

11. Rao P and Segal BM: Experimental autoimmune encephalomyelitis. Methods Mol Med 102: 363-375, 2004.

12. Steinman L: Antigen-specific therapy of multiple sclerosis: The long-sought magic bullet. Neurotherapeutics 4: 661-665, 2007.

13. Oukka M: Interplay between pathogenic Th17 and regulatory T cells. Ann Rheum Dis 66 (Suppl 3): iii87-iii90, 2007.

14. EdwardsLJ,Robins RA and Constantinescu CS:Th17/Th1 phenotype in demyelinating disease. Cytokine 50: 19-23, 2010

15. Benson JM, Stuckman SS, Cox KL, Wardrop RM, Gienapp IE, Cross AH, Trotter JL and Whitacre CC: Oral administration of myelin basic protein is superior to myelin in suppressing established relapsing experimental autoimmune encephalomyelitis. J Immunol 162: 624754, 1999.
16. Qu N, Zhou XY, Han L, Wang L, Xu JX, Zhang T, Chu J, Chen Q, Wang JZ, Zhang Q and Tian Q: Combination of PPT with $\mathrm{LiCl}$ treatment prevented bilateral ovariectomy-induced hippocampal-dependent cognition deficit in rats. Mol Neurobiol Dec 23 [Epub ahead of print], 2014.

17. Lehuen A, Diana J, Zaccone P and Cooke A: Immune cell crosstalk in type 1 diabetes. Nat Rev Immunol 10: 501-513, 2010.

18. Lisak RP: Neurodegeneration in multiple sclerosis: Defining the problem. Neurology 68: S5-S12; discussion S43-S54, 2007

19. Goverman J: Autoimmune T cell responses in the central nervous system. Nat Rev Immunol 9: 393-407, 2009.

20. Miller AH, Maletic V and Raison CL: Inflammation and its discontents: The role of cytokines in the pathophysiology of major depression. Biol Psychiatry 65: 732-741, 2009

21. Murphy AC, Lalor SJ, Lynch MA and Mills KH: Infiltration of Th1 and Th17 cells and activation of microglia in the CNS during the course of experimental autoimmune encephalomyelitis. Brain Behav Immun 24: 641-651, 2010.

22. Friese MA and Fugger L: Pathogenic CD8(+) T cells in multiple sclerosis. Ann Neurol 66: 132-141, 2009.

23. Fletcher JM, Lalor SJ, Sweeney CM, Tubridy N and Mills KH: $\mathrm{T}$ cells in multiple sclerosis and experimental autoimmune encephalomyelitis. Clin Exp Immunol 162, 1-11, 2010.

24. Saxena A, Martin-Blondel G, Mars LT and Liblau RS: Role of CD8 $\mathrm{T}$ cell subsets in the pathogenesis of multiple sclerosis. FEBS Lett 585, 3758-3763, 2011.

25. Sobottka B, Harrer MD, Ziegler U, Fischer K, Wiendl H, Hünig T, Becher B and Goebels N: Collateral bystander damage by myelin-directed CD8+ T cells causes axonal loss. Am J Pathol 175, 1160-1166, 2009.

26. Niederkorn JY: Emerging concepts in CD8(+) T regulatory cells. Curr Opin Immunol 20: 327-331, 2008. 